\title{
VII.
}

\section{Beiträge zur Anatomie des kindlichen Schläfenbeines.}

\author{
Von \\ Dr. G. J. Wagenhltuser \\ in Wârzburg. \\ (Hierzu Taf. I-III.) \\ I. \\ Ueber die Fossa subarcuata.
}

Entwicklung und topographische Verhältnisse mit vergleichendanatomischen Betrachtungen.

An der hinteren Fläche der Felsenbeinpyramide des Erwachsenen, seitwärts vom Porus acust. intern. und näher dem oberen Rande, findet sich in den Lehrbiichern der Anatomie eine narbig eingezogene, von der Spitze der Pyramide her zugängliche Vertiefung angegeben, eine unvollständig ausgefültte Grube unter dem oberen verticalen Bogengange als Ueberbleibsel einer am embryonalen Felsenbeine unter der Wölbung des oberen Canalis semicircul. befindlichen Grube, welche v. Tröltsch als Fossa subarcuata bezeichnet hat.

In dieser feinen, nach Henle blindendigenden Vertiefung, Hiatus subarcuatus von v. Tröltschi), Canalis petroso-mastoideus von Voltolini ${ }^{2}$ ) genannt, verläuft, wie die genannten Autoren hervorheben, durch die Knochenmasse hindureh bis in das Antrum mastoideum hin ein bindegewebiger Gefässstrang der Dura mater, eine Falte, die wahrscheinlich ein Gefäss enthält nach Voltolini, den Rest einer dicken Falte, eines starken Gefässfortsatzes der Dura mater bildend, der beim Embryo und Neugeborenen unter dem Can. semicircul. sup. eine tiefe Grube hier bedingend, in die Tiefe des Felsenbeinknochens eindringt

1) v. Tröltsch, Arch. f. Ohrenheilk. IV. Bd. S. 128.

2) Voltolini, Virchow's Archiv. XXXI. Bd. S. 202.

Archiv f. Ohrenheilkunde. XIX. Ba. 
bis in die Gegend hin, wo sich später die Mastoidzellen entwickeln.

Auf den Gefässinhalt der genannten Vertiefung im Felsenbeine des Erwachsenen machen von den Anatomen nur $\mathrm{K} \mathrm{rause}^{1}$ ) und $A \operatorname{rnold}^{2}$ ) aufmerksam, indem ersterer dieselbe als Canalis diploicus bezeichnet, und letzterer anfuhrt, dass hier eine Arterie und Vene in und aus der Knochensubstanz trete.

Dass diese Oeffnung mit dem Inhalte, den sie umschliesst, für den Praktiker von Bedeutung werden kann, haben mehrere Fälle von Odenius, Voltolini, v. Tröltsch, sowie ein vierter, den Hartmann ${ }^{3}$ ) aus seiner eigenen Sammlung beschrieben anfuhrt, bewiesen; Fälle, in denen eine Ueberleitung eines entzïndlichen Processes vom Inneren des Schläfenbeines nach dem Inneren der Schädelhöhle zur Dura mater $\mu$ nd dem Sinus petrosus sup. auf diesem Wege stattfand.

Wäbrend bisher nur in der angegebenen Richtung, also in der Verbreitung entzündlicher Processe aus dem Inneren des Schläfenbeines zur Schädelhöhle, die Bedeutung dieser Bildung gesucht wurde, weist ihr in der neuesten Zeit $L \mathrm{uca}^{4}$ ) in seiner für die Betrachtung der Labyrinthaffectionen nene Gesichtspunkte und Perspectiven eröffnenden Arbeit über "Hämorrhagie und bämorrhagische Entzïndung des kindlichen Ohrlabyrinths “, namentlich für das kindliche Alter, eine verhängnissvolle Wichtigkeit $z u$, indem ex nachwies, wie es auf diesem Wege durch die gerade hier, beim Kinde, so häufigen Erkrankungen der benachbarten Schädel- und, wie wir später sehen werden, auch der Trommelhöble zu consecutiven Ernährungsstörungen der Pyramide und des Labyrinthes selbst kommen kann.

Die tiefe, unter dem Can. semicircul. sup. des Fötus und Nengeborenen befindliche Grube, welche uns hier beschäftigen soll und auf welche v. Tröltsch wiederum die Aufmerksamkeit gelenkt hat, ist bereits lange bekannt. Schon Duverney beschreibt dieselbe im Capitel über die Structur des Ohres beim

1) W. Krause, Handb. d. Anatomie d. Menschen. 1843. I. S. 244.

2) Arnold, Ebenda. 1845. I. S. 397.

3) Odenius, Medicinsk. Archiv. III. Bd. Nr. 4. - Voltolini und v. Tröltseh a. a. O. - Hartmann, Die Krankh. d. Ohres u. deren Behandlung. 1881. S. 140 .

4) A. Lucae, Virchow's Archiv. 88. Ba. Heft 3 .

5) Franc Duverney, Tractatus de organo auditus. Uebersetzt von J. A. Mischel. Berlin 1732. S. 52 . 
Fötus und bildet sie auf Taf. XV, Fig. 3 , ab, ebenso findet sie später noch häufig Erwähnung. Nur über die Deutung ihres Inhaltes erweisen sich die Ansichten nicht völlig ubereinstimmend. Huschke ${ }^{1}$ ) bezeichnet dieselbe beim Neugeborenen als ,einen grossen, mit harter Hirnhant ausgefullten Trichter ", in gleicher Weise nennt sie Sappey ${ }^{2}$ ) "un trou considérable comblé per la dure mère", Voltolin $i^{3}$ ) von einer dicken, sulzigen, gallertigen Falte der Dura mater", und Henle ${ }^{4}$ ) von Knorpel ausgefüllt. Nach v. Tröltseh (1. c.) enthält dieselbe ein dickwandiges, sogleich nach allen Seiten Aestchen abgebendes Blutgẹfäss, und zwar eine dickwandige, an elastischen Elementen besonders reiche Arterie, während $O$ deni u s (l. c.) sich für die venöse Natur dieses Gefässes ausspricht. Neben dieser Arterie sah v. Tröltsch allerdings zuweilen eine weit feinere Vene verlaufen, deren Einmündung in den Sinus petrosus sup. er zweimal nachweisen konnte. Er bezeichnet dieses, einer Arteria nutritiae gleichstehende Gefäss, welches "für die Entwicklung des Schläfenknochens jedenfalls von Wichtigkeit sein kann " (Lehrbuch 7 . Aufl. S. 210), als Arteria subarcuata und die Vene (eine Vena diploica) als Vena subarcuata. Ueber die Grube selbst gibt er an, dass dieselbe beim fünfmonatlichen Fötus $7 \mathrm{Mm}$. hoch und $5 \mathrm{Mm}$. breit sei und durch das ganze knöcherne Felsenbein und dessen Basis hindurch gehe und hinter der Ohrmuschel, und zwar mit ziemlich grosser zackiger und unregelmässiger Oeffnung an der Aussenfläche des Kopfes, von der Haut nur durch eine diinne Knorpellage getrennt, münde. Noch beim Neugeborenen $5 \mathrm{Mm}$. hoch and $4 \mathrm{Mm}$. breit, mündet sie, in der Mitte schlitzförmig verengt, an der Aussenfläche des späteren Warzenfortsatzes unter und in seinem Knorpelüberzuge.

Verfolgt man die Entwicklung dieser Grube in den verschiedenen embryonalen Perioden, so findet sich eine erste Andeutung derselben zu einer Zeit, wo die Schädelbasis knorpelig geworden ist. Zu dieser Zeit (2. und 3. Monat) zeigt die knorpelige, mehr birnförmig gestaltete und nach hinten kugelig endende Pars petrosa des Felsenbeines hinter dem Meatus auditorius internus eine seichte, rundliche, in der Tiefe durch Knorpel verschlossene

1) H uschke, Sömmering, Lehre von d. Eingeweiden u. Sinnesorganen. 1844. S. 900 .

2) Sappey, Traité d'anatomie descriptive. Tome 1. p. 158.

3) Voltolini, Monatsschrift f. Ohrenheilk. 1868. S. 22.

4) Henle, Knochenlebre. 1871. S. 167. 
Grube von ungefähr gleichem Durchmesser wie jener. Kölliker hat dieselbe in seiner Entwicklungsgeschichte (2. Auf. Leipzig 1879) in den Figuren 266, 280 und 281 abgebildet.

Ein Präparat ( $31 / 2-4$ Monate), an dem bereits die Schädelbasis an der Pars basilaris, condyloid. und squamosa ossis occipitis, sowie an den beiden Keilbeinen Knochenpunkte zeigt, die Aussenfläche des Schädels an der Squama ossis petrosi und am Annulus tympanicus Verknöcherung aufweist, lässt die Pyramide in veränderter Form erscheinen.' Das hintere und äussere Ende derselben. hat sich über das Niveau der übrigen Pyramidenfläche bedeutend erhoben und bildet einen fast senkrecht auf der Axe der Pyramide stehenden Bogen, unter dem eine rundliche, $1 \mathrm{Mm}$. weite Oeffnung in eine ebenso weite und tiefe Höhlung führt. Dieser Bogen stösst nach hinten und aussen hart an die knorpelige Parietalplatte an, fast unmittelbar in dieselbe tibergehend (s. Fig. 1, Taf. I).

Ein weiteres Präparat, an welchem die ganze Pyramide schon verknöchert erscheint $\left(5^{1 / 2}-6\right.$ Monat), zeigt die vorbeschriebene Form der Felsenbeinpyramide in ausgesprochenerem Grade. Der obere halbcirkelförmige Kanal ragt in Form eines knöchernen Bogens, in seinen zwei oberen Dritttheilen vollständig frei und senkrecht auf der Pyramidenaxe stehend, hervor, eine $2 \mathrm{Mm}$. weite, querliegende, länglichrunde Oeffnung, die Fossa subarcuata, umschliessend. Der Raum nach hinten und aussen von diesem Bogen, wohin diese Oeffnung fuihrt, die spätere Pars mastoidea des Schläfenbeines, wird von Knorpelmasse ausgeftillt, in der zwei Knochenkerne sichtbar sind, ein vorderer und ein grösserer hinterer, welch letzterer bereits mit dem Bogen des äusseren, halbkreisförmigen Randes verschmolzen ist. Die hintere Umrandung des knöchernen Can. semicircul. sup. liegt der knorpeligen Pars parietalis noch ungemein nahe, durch einen $11 / 2$ bis $2 \mathrm{Mm}$. breiten, knorpeligen Saum getrennt (Fig. 2, Taf. I).

In einer weiteren Periode der Entwicklung (6. und 7. Monat) hat sich mit der Grössenzunahme des Can. semicircul. sup., sowie durch Ablagerung von Knochenmasse an dessen ganzer hinterer Umrandung und Verschmelzung derselben mit den gleichfalls vereinigten Knochenkernen der Pars mastoidea der unter dem oberen halbkreisförmigen Kanal gelegene Hohlraum wesentlich vergrössert, so dass derselbe nunmehr einen rings von Knochen umschlossenen, $7 \mathrm{Mm}$. weiten und $5 \mathrm{Mm}$. tiefen, das Felsenbein durchsetzenden Kanal darstellt, welcher gegen die Aussenfläche 
des Schädels zuftihrt und hier mit $5 \mathrm{Mm}$. weiter, von Knorpelmasse umschlossener Oeffnung mündet. Durch die an der hinteren Umrandung des Can. semicircul. sup. erfolgte Knochenanlagerung hat sich die Entfernung desselben von der Schädelwand bedeutend vergrössert, so dass dieselbe nunmehr $6 \mathrm{Mm}$. beträgt und sich zugleich zwei gegen das Cerebrum und Cerebellum zugelagerte Wände oder Flächen des Felsenbeines gebildet haben (Fig. 3, Taf. I).

Beim weiteren Wachsthume des Felsenbeines, wobei vorgenannter Abstand des Can. semicircul. sup. von der Schädelwand ungemein rasch zunimmt, so dass derselbe zu Ende des 8. Monats 10-12 Mm. beträgt, tritt eine weitere Grössenzunahme der Fossa subarcuata nicht mehr ein. Sie führt, eine Strecke lang von gleicher Weite wie am Eingange, manchmal eher sich noch etwas vertiefend, rings von Knochenmasse umgeben, bis an die äussere Schädelfläche, wo die rundliche Oeffnung, bedeutend verengt, durch Knorpel verschlossen erscheint. Beim Neugeborenen hat sich der Umfang derselben schon wieder etwas verkleinert, hat jedoch immer noch eine Höhe und Breite von $5 \mathrm{Mm}$. und fuhrt dann als enger Kanal entweder an die Anssenfläehe des Schädèls, wo dann noch nach oben zu eine kleine, nur durch Knorpel geschlossene Stelle sichtbar ist, oder mündet, wenn die Verknöcherung an der Aussenfläche der Pars mastoidea vollständig stattgefunden hat, als ein feiner, immerhin $1 \mathrm{Mm}$. weiter Kanal an der hinteren Fläche der Felsenbeinpyramide (Fig. 4 u. 5, Taf. I).

Was die Dauer dieser Grube anbelangt, so findet sich dieselbe in ihrer urspriinglichen Form als rundliches Loch, wenn auch bedeutend verengt, bis gegen das 3.-5. Lebensjahr hin vor. In den späteren Jahren verwandelt sich dieselbe in einen länglichen Spalt, der bis zum 8. und 10. Lebensjahre in ziemlicher Weite erbalten bleiben kann; in ihren Zügen, als Hiatus subarcuatus, erhält sich dieselbe in der gleich zu Anfang geschilderten Weise als constante Bildung das ganze Leben hindurch. Als Inhalt der Fossa subarcuata lässt sich beim Embryo in den letzten Fötalmonaten, sowie in den ersten Lebensjahren des Kindes durch Zug ein zapfenförmiges Gebilde entfernen, das sich bei makroskopischer Betrachtung als eine Fortsetzung der Dura mater darstellt und genau die Form des Hohlraumes zeigt. Anfänglich einen kolbigen, 5-6 Mm. langen und ebenso dicken Zapfen beim Neugeborenen bildend, verjüngt es sich dann weiter nach aussen zu und endet meist gabelförmig getheilt 
mit zwei Aestchen. Die Gesammtlänge dieses Fortsatzes ist eine ziemlich beträchtliche, zwischen 10 und.16 $\mathrm{Mm}$. betragend. Auch beim Erwachsenen noch sieht man beim Abziehen der Dura einen, wenn auch bedeutend dunneren, Fortsatz derselben in den genannten Spalt hinein sich erstrecken.

\section{Topographische Verhältnisse der Fossa subarcuata.}

Zur weiteren Untersuchung der Fossa subarcuata, namentlich in Bezug auf Inhalt, Verlauf und topographische Verhältnisse habe ich es unternommen, eine Anzahl von Serienschnitten durch das Felsenbein verschiedenalteriger embryonaler und kindlicher Exemplare herzustellen. Die betreffenden Präparate wurden zum Theil mit Müller'scher Flüssigkeit und Salpetersäure, theils mit 5 proc. Salpetersäure allein entkalt, gewässert und in Alkohol erhärtet, um nach dem Schneiden dann entweder ungefärbt oder nach Färbung mit Pikrocarminlösung zur Untersuchung verwendet zu werden.

Die erste dieser Serien ist von einem Präparate aus der Zeit zwischen 5. und 6. Fötalmonate gewonnen. Auf den senkrecht zur Axe des Felsenbeines gelegten Durchschnitten sehen wir, wie die Uura mater sich in die Höblung unter dem Can. semicircul. sup, hineinerstreckt, wie dann weiter nach aussen dieser Fortsatz derselben in der Knorpelmasse erscheint, die den Raum zwischen den Durchschnitten der knöchernen Bogengänge ausfullt, und sich durch seine bindegewebige Structur deutlich von der umgebenden Knorpelmasse abhebt. Zugleich lässt sich erkennen, dass derselbe Gefässe enthält, in der Mitte desselben erscheint der Durchschnitt eines solchen, und sieht man allenthalben gegen die knöcherne Umgebung der Bogengänge gefässhaltige Bindegewebszüge verlaufen. Weiter nach aussen zu erscheint derselbe dann bedeutend verschmächtigt, rings von Knorpelmasse umgeben und zahlreiche Ausläufer in sie hinein ausschickend und verschwindet er dann allmählich völlig in dem Knorpelgewebe (Fig. 1-3, Taf. II).

Auf Schnitten rom Felsenbeine älterer Embryonen sehen wir insofern eine Aenderung im Verhalten dieses Duralfortsatzes, als derselbe mächtiger erscheint und den ganzen Raum zwischen den Durchschnitten der knöchernen Bogengänge einnimmt. Derselbe zeigt sich wiederum gefässhaltig und sendet aller Orten Gefässzweige in das weitmaschige Knochengewebe um die Bogen- 
gänge herum. Weiter nach aussen zu verliert er sich dann wieder gegen das daselbst noch restirende Knorpelgewebe zu.

Die gleichen Verhältnisse stellen sich dann beim Neugeborenen und in den ersten Monaten des kindlichen Alters dar. Auf einem, das Felsenbein gleichfalls senkrecht zu seiner Axe treffenden und vor der vorderen Umrandung des Can. semicircul. sup. gelegenen Schnitt erkennt man . deutlich, wie die Dura mater sich zur Ausbuchtung der Fossa subarcuata verbält. Man sieht, wie in den Fasermassen der Dura eine verschiedene Richtung: eintritt; die einen, und zwar die oberflächlichen Fasern, behalten ihre Richtung bei und streichen darüber hinweg, während die tieferen umbiegen und, der Umrandung des Knochens folgend, in dieselbe eindringen. Dabei zeigt sich nach aussen zu die schichtenweise Anordnung noch erhalten, während gegen die Mitte $\mathrm{zu}$ ein verschlungener und sich kreuzender Faserlauf eintritt. So ziemlich in der Mitte der eindringenden bindegewebigen Masse erscheint der Durchschnitt eines grösseren Gefässes, einer dünnwandigen Vene, an der regelmässig wandständig gelagert eine kleinere dickwandigere und deutlich von der Vene sich unterscheidende Arterie sich befindet. Zahlreiche Gefässdurchschnitte lassen sich sodann noch in der Umgebung der in der Mitte gelegenen Gefässe, sowie gegen die Umrandung des Knochens hin nachweisen.

Auf den Schnitten weiter nach aussen zu finden wir bei ganz demselben Verhalten in der Structur der uns beschäftigenden Bildung jetzt die Durchschnitte der verschiedenen Bogengänge die Umrandung derselben bilden. So erscheint nach oben der frontale, nach hinten der sagittale und nach unten und aussen zu der horizontale Kanal, nach der Oertlichkeit des Schnittes, in verschiedener Ausdehnung an der Umgrenzung betheiligt, nur durch mehr oder minder breite Streifen weitmaschigen Knochengewebes, in welche Maschen sich zahlreiche bindegewebige und gefässhaltige Aeste verfolgen lassen, von dem Bindegewebs- und Gefässstrange getrennt.

Noch weiter nach aussen zu fehlen jedoch diese Durchschnitte der Kanäle, nur der hintere lässt sich noch etwas weiter verfolgen und tritt jetzt ein anderes nachbarliches herdialtidss ein, es erscheint nach unten zu die erste Andeupnt der Hơr räume des Warzenfortsatzes, das dem betreffendons thérontsprechend erweiterte Antrum Valsalvae. Eine nicht solư breit schicht weitmaschigen Knochengewebes trennt den Bipidegewebstrif 
von dem Antrum, zu dem mächtige gefässhaltige Züge von jenem her verlaufen, niemals jedoch bis an die Auskleidung desselben gelangen, sondern in den Maschen des Knochengewebes verschwinden. Der Bindegewebsstrang selbst lässt sich noch eine Zeit lang, bedeutend verschmälert und meist in zwei Aeste getheilt, immer aber noch deutliche Gefässdurchschnitte zeigend, verfolgen, bis er sich nach ąussen zu dann völlig in der Spongiosa des Knochens verliert (Fig. 3-6, Taf. II).

An den Schnittserien älterer kindlicher Individuen $(21 / 2,3$ und 4 Jahren) zeigten sich dieselben Verhältnisse, nur dass einmal der eindringende gefässhaltige Bindegewebsstrang, entsprechend der verkleinerten Eintrittsöffnung, an Umfang geringer sich erwies und dann nicht so weit nach aussen hin verfolgt werden konnte, immer jedoch bis in die Gegend hin, wo sich im Warzenfortsatze die Hohlräume der Warzenzellen zu entwickeln beginnen.

Wie hochgradig sich in pathologischen Fällen der Blutreichthum der uns beschäftigenden Bildung gestalten kann, erwiesen Schnitte durch das Felsenbein eines $11 / 2$ jährigen, an Meningitis basilaris gestorbenen Kindes. Das Gefäss in der Mitte erschien strotzend gefüllt, umgeben von einem Netze kleinerer und kleinster Aeste, namentlich zeigte sich die knöcherne Umrandung von einem ganzen Gefässkranze eingefasst, von dem aus aller Orten mächtige Zweige in den Knochen eindrangen.

Die mikroskopische Untersuchung bietet, wie wir selien, eine Bestätigung und Ergäuzung der makroskopischen Beobachtungen dar, indem sie uns den Verlauf dieses von der Dura abzweigenden Fortsatzes in den verschiedenen Regionen des Felsenbeines erkennen lässt und zugleich zeigt, dass derselbe gefässhaltig ist und zwar, wie meine Präparate constant ergeben, eine grọsssere Vene und eine kleinere Arterie enthält. Eine Einmündung der Vene in den Sinus petrosus sup. konnte ich, da an dieser Stelle die Dura meist bei der Präparation sich abgelöst hatte, nicht nachweisen. Weiter vermag diese Untersuchung die praktische Wichtigkeit der uns beschäftigenden Bildung deutlich zu illustriren, indem sie die nachbarlichen Beziehungen derselben zu den knöchernen Bogengängen, den Bindegewebs- und Gefässzusammenhang mit denselben uns vor Augen führt, was, wie schon hervorgehoben, von Lucae in neuester Zeit besonders betont wurde, und sodann noch darthut, wie dieselbe in dem Knochengewebe, das um die Hohlräume des Warzenfortsatzes herum sich vorfindet, zur Ausbreitung kommt, so dass entzünd- 
liche Processe in jenen ein Mitergriffenwerden derselben und eine Weiterverbreitung auf diesem Wege hervorrufen können, welchen Vorgang die friiher genannten Autoren zu beobachten Gelegenheit hatten.

Lucae macht in der angegebenen Arbeit zu wiederholten Malen auf das nahe Herantreten der Spongiosa an die Bogengänge, auf enorm weite und langgestreckte, in unmittelbarster Nähe der letzteren befindliche Knochenmaschen aufmerksam, welchen er, in Gemeinschaft mit den von ibnen aus zur Innenwand der Kanäle dringenden Gefässen, für die Ueberleitung entzündlicher Processe hohe Wichtigkeit beilegt. Ich habe darauf hin den betreffenden Verhältnissen gleichfalls meine Aufmerksamkeit zugewendet und fand an meinen anatomischen Präparaten diese Angaben Lucae's vollständig bestätigt. Beim Embryo in den letzten Monaten und auch noch beim Neugeborenen werden diese weiten Maschen der Spongiosa nur durch eine schmale Schicht compacten knöchernen Gewebes von dem Innenraume der Kanäle geschieden (wie dies auch schon bei der makroskopischen Betrachtung sich ergibt, wo diese Schicht kaum von der Dicke des feinsten Postpapieres sich erweist). Im weiteren Verlaufe der Entwicklung nimmt sie, unter Verdrängung des spongiösen Gewebes, bedeutend an Mächtigkeit zu, erweist sich jedoch immer von weiten und zahlreichen, sich verästelnden Gefässen durchsetzt, die sich (auch an den Schnitten durch das Felsenbein des 4 jährigen Kindes war dies noch deutlich zu erkennen) von diesen Knochenmaschen aus bis zum Lumen der Kanäle verfolgen liessen.

Ueber die Entwicklung der Fossa subarcuata gibt Kölliker (Entwicklungsgeschichte S. 741), der, wie ich einer Mittheilung: von ihm verdanke, gleichfalls Schnitte durch dieselbe gemacht hat, an, dass er dieselbe anfänglich in der Tiefe durch Knorpel geschlossen gefunden habe. Später jedoch entwickelten sich in der Cartilago petrosa in dieser Gegend Gefässe und im Inneren Höhlungen, so dass dann bei der Verknöcherung in der That, wie v. Trölts ch angibt, ein durchgehender Kanal entsteht, der von einem gefässhaltigen Bindegewebsstrange erftillt ist.

\section{Bedeutung der Fossa subarcuata.}

Betrachten wir die Fossa subarcuata in den letzten Fötalmonaten, so muss sie uns durch ihre Grösse und Mächtigkeit imposant und auffällig erscheinen, wie sie auch von Kölliker 
(a. a. 0. S. 741) als eine auffallende Bildung an der embryonalen Cartilago petrosa schon bezeichnet wird. Bei einer Umschan in der einschlägigen Literatur nach erklärenden Angaben über dieselbe scheinen wir lediglich auf das, was v. Tröltseh und neuerdings Lucae anfithrt, beschränkt. Beide fassen dieselbe als Ernährungsloch auf und sehreiben ihr eine Bedeutung für die Entwicklung des Schläfenknochens und besonders des um die Kanäle sich anlagernden Knochengewebes zu. Zugleich macht ersterer auf eine ähnliche, constant unter dem hinteren Bogengange sich vorfindende Bildung aufmerksam, welche allerdings nie so bedeutend entwickelt sich zeigte.

Wenn auch das Ergebniss der mikroskopischen Untersuchung: sich mit dieser Deutung vollständig in Uebereinstimmung bringen lảsst und auch der Umstand, dass zur Zeit, wo das Wachsthum und die Grössenzunahme des Knochens ein gegenïber dem Wachsthume in den fötalen Perioden geringeres und langsameres wird, diese Bildung an Umfang und Mächtigkeit verliert und zur Zeit der vollen Ausbildung des Knochens eine gegenüber dem embryonalen Zustande geradezu minimale Bedeutung besitzt, als hierfür sprechend angesehen werden kann, so geniugt diese Deutung doch nicht vollständig; auffallend muss immerhin hierbei die für ein Ernährungsloch so grosse Weite und Mächtigkeit bleiben und der Contrast, in dem dieselben zur Grösse des eindringenden Gefässes stehen. Zugleich wäre es als das einzige bestimmte und constante Ernährungsloch von diesem Umfange an den Knochen des Schädels aufzufassen.

$\mathrm{Zu}$ einer wesentlich anderen Auffassung dieser Bildung musste eine Beobachtung hinleiten, die sich bei $v$. Trölts $\mathrm{ch}^{1}$ ) in einer Arbeit, "Beiträge zur vergleichenden Anatomie der Obrtrompete ", vorfindet. Er macht daselbst auf eine beim Hasen vorkommende, 4-5 Mm. im Durchmesser haltende rundliche Vertiefung im Knochen aufmerksam, welche sich über und hinter dem Porus acust. intern. findet. $n$ Dieselbe, ganz analog erscheinend jener constanten, ein Blutgefäss enthaltenden Vertiefung, die sich beim menschlichen Fötus unter dem Canalis semicircul. sup. findet, ist mit Gehimmasse ausgefillt und geht so tief in das Felsenbein hinein, dass sie nur durch eine durchscheinend düne Knochenplatte von der Aussenfläche des Schädels getrennt ist."

Nachdem nun noch gleich zu Anfang der Befund am Felsenbein eines jungen Affen, das ich der Freundlichkeit des Herrn

1) v. Tröltsch, Arch. f. Ohrenheilk. II. Bd. S. 225. 
Prosector Dr. Ph. Stöhr verdanke, mein Interesse erweckt hatte, wo sich nämlich tiber und hinter dem Meatus auditorius intern. und unter dem deutlich durchscheinenden Canalis semicircul. sup. der $5 \mathrm{Mm}$. hohe, rundliche Eingang zu einem sich noch erweiternden, $6 \mathrm{Mm}$. tiefen Hoblraum zeigte (Fig. 6, Taf. I), der von der. Dura mater ausgekleidet und von Gehirnmasse erfüllt war, lag die Aufforderung ungemein nahe, weitere Umschau zu halten, wie es mit dem Vorkommen einer solchen Bildung am Felsenbeine in den verschiedenen Thiergattungen, sowohl im ausgewachsenen als embryonalen Zustande, aussieht, um vielleicht hieraus dann einen Schluss auf die Bedeutung derselben beim Menschen ziehen zu können.

Die Lehrbücher der vergleichenden Anatomie boten, an zahlreichen Stellen zerstreut, Andeutungen hieriber dar. Am weitesten zurückgehende Aufzeichnungen über diese Bildung fanden sich bei $\mathrm{Cuvier^{1 } )}$ bereits in der ersten Auflage vor. Er sagt in der VIII. Leçon (Ostéologie de la tête) bei der Besprechung des inneren Gehörganges: „Dans les singes, au dessus et en dehors du conduit auditif interne il-y-a un enfoncement plus large que lui, et non percé dans son fond, qui sert à loger une protubérance du cérébel. Il manque dans l'orang-outang et dans le jocko.

Dans les carnassiers l'enfoncement aveugle est plus profond encore que dans les singes."

Bei $\mathrm{Meckel}{ }^{2}$ ) lassen sich keine auf die uns beschäftigende Bildung bezïgliche Angaben nachweisen, ebensowenig findet dieselbe bei Blumenbach ${ }^{3}$ ) irgend welche Beruicksichtigung.

Ausfïhrliche Erwähnung, und zwar durch die ganze Reihe der Sängethiere hindurch, findet dieselbe sodann bei $\mathrm{Cuvier}{ }^{4}$ ) in der zweiten Auflage. Er bezeichnet sie hinfort als „Enfoncement cérébelleux" und führt sie bei allen Affen (von dem Gebbon $a b$ ) auf. Weniger tief bei den Kynocephalen, sei sie am mächtigsten beim Faulthierlori.

Von den Fleischfressern filhrt er Igel, Tenrek, Chrysochloris, Hund, Bär, Nasen und Zibeththier, Katze und Stinkthier an VIII.

1) G. Cuvier, Leçons d'Anatomie comparée. Tome II. p. 54. Paris

2) J. F. Meckel, System d. vergleichend. Anatomie. Halle 1825.

3) J. F. Blumenbach, Geschichte und Beschreibung der Knochen. Göttingen 1807.

4) G. Cu vier, Leçons d'Anatomie comparée publiées par M. Duméril. Seconde édition. Paris 1837. Tome II. 
auf, wo sie nur klein ist, während sie beim Maulwurf, wo sie zugleich mit einer rundlichen Oeffnung an der Aussenfläche des Schädels mündet, beim Dachs, Wiesel und Marder hoch entwickelt sich zeigt. Von den Robben erscheint sie beim gemeinen Seehund ungemein gross und geräumig, während sie bei den Ohrenrobben vollständig fehlt.

Unter den Beutelthieren zeigen sie als tiefe Grube das Beutelthier (Sarigue) und der Beuteldachs. Beim Hypsiprymnus oder Potoroo mïndet sie ähnlich wie beim Maulwurf an der äusseren Schädelfläche.

Aus der Ordnung der $\mathrm{N}$ ager werden mit stark entwickelter Grube angefiuhrt: Der Aye-Aye, Hase, Murmelthier, Biber, Ratte, Maus, Hamster, Gerbille; angedeutet nur als leichte Einsenkung beim Stachel- und Meerschweinchen, vollständig fehlend bei der brasilianischen Ratte (Paca).

Unter den Edentaten fehlt sie vollständig mit Ausnahme von Tamandua und Fourmilier didactyla. Gleichfalls vollständig wird dieselbe vermisst bei allen Dickhäutern, Wiederkänern und Walen.

Unter den Monotremen erwähnt Cuvier des Ornithorhynchus (Schnabelotter), bei welchem die halbcirkelförmigen Kanäle stark nach innen vorspringen und ein wohlmarkirtes Kreuz bilden, „et qui paraît bien l'analoque de l'enfoncement cérébelleux que nous avons rencontré dans beaucoup d'animaux. " Die einzige Stelle, an welcher $\mathrm{Cu}$ vier auf das nachbarliche Verhältniss zwischen Bogengängen und dieser Bildung hinweist.

Köstlin's ') Angaben uiber diese Grube bilden nur eine unvollständige Wiedergabe des schon von $\mathrm{Cuvier}$ Gesagten.

Eine weitere Untersuchung, und zwar gleichfalls durch die ganze Säugethierreihe, erfuhr diese Bildung durch $\mathrm{Hyrt} \mathrm{r}^{2}$ ) Der III. Abschnitt (Labyrinth) seiner vergleichend anatomischen Untersuchungen enthält die Ergebnisse derselben. Auch er fand diese Grube bei allen Gattungen der Quadrumanen, relativ am grössten beim Faulthierlori aus Bengalen. Bei einem sehr jungen Exemplare von Simia satyrus war dieselbe vorbanden, an einem alten von S. satyrus und troglodytes dagegen verschwunden.

Bei den Cheiropteren allgemein vorkommend, erreicht

1) O. Köstlin, Der Bau des knöchernen Kopfes. 1844. $\$ 34$ u. ff.

2) J. Hyrtl, Vergleichend anatomische Untersuchungen über d. innere Gehörorgan des Menschen u. d. Säugethiere. Prag. 1845. S. 101. 
sie ihre grösste Entwicklung beim Flattermaki (Galeopithecus volans).

Unter den Carnivoren fehlt sie nach ihm bei den Gattungen Felis und Canis.

Bei den Nagern soll sich dieselbe am schönsten ausgesprochen zeigen bei allen kletternden, hüpfenden und wühlenden Nagern.

Seine übrigen Angaben stimmen völlig mit den von $\mathrm{Cuvier}$ gemachten uberein. Das Gesammtresultat seiner Untersuchung stellt er in folgenden Sätzen zusammen: Der obere Canalis semicircularis umgibt bei vielen Säugethieren den Eingang einer Höhle, die den Raum zwischen den drei Bogengängen einnimmt und entweder durch das innere Blatt der Dura mater von der Schädelböhle getrennt wird, während das äussere als tauber Beutel sich in sie hineinstiilpt, oder von einer Ausstiulpung beider Blätter derselben Membran ausgekleidet wird und gewisse flockenartig vereinigte Läppchen des kleinen Gehirns aufnimmt. Auch im embryonalen Schläfeknochen des Menschen existirt diese Höhle, verschwindet aber mit der vorschreitenden Entwicklung des Felsenbeines und lässt nur ausnabmsweise eine narbig eingezogene Knochenfissur zurück, die der Stelle des früheren Einganges entspricht."

Unter den neueren Autoren finden wir bei $O \mathrm{wen}^{1}$ ) und Huxle ${ }^{2}$ ) eine Erwähnung der genannten Grube. Die Angaben, die ersterer iber "the cerebellar fossa on the upper part of the petrosal " macht, bestätigen vollständig das bereits von $\mathrm{Cuvier}$ Gesagte, während letzterer dieselbe nur zweimal aufführt, bei der Phoca und bei der Gattung Ateles, wo er sagt: „Auf der Innenseite des Ohrknochens ist eine Grube, welche vom vorderen und senkrechten halbkreisförmigen Kanal überwölbt wird und in welche der Flocculus zu liegen kommt."

Zur Pruffung der von den genannten Autoren gemachten Beobachtungen, so weit eine solche mir tiberhaupt möglich sein konnte, habe ich die leichter zu verschaffenden Thiere in frischem Zustande, oder sagittal durchschnittene oder gesprengte Schädel aus der Sammlung des zootomischen Institutes benutzt, an kostbaren und nicht weiter zu zerlegenden Exemplaren mittelst Spiegels

1) R. Owen, On the Anatomy of Vertebrates. London 1866. Vol. II. S. 347 u. ff.

2) T. H. Huxley, Handbuch d. Anatomie d. Wirbelthiere. Uebersetzt von Ratzel. Breslau 1873. S. 358 und 389. 
und Reflector durch das Hinterhauptloch hindurch eine Besichtigung ermöglicht.

Bezüglich des Fehlens der genannten Höhle bei der Gattung: Felis ergab die Untersuchung einen Widerspruch mit der Angabe Hyrtl's. An einem gesprengten Katzenschädel der Sammlung findet sich die fragliche Grube in ziemlicher Tiefe vor, ebenso lässt sich dieselbe, wenn auch als seichtere Einsenkung, bei der Wildkatze, Löwe und Panther nachweisen.

In gleicher Weise verhält es sich bezüglich der Gattung Canis. In frischem Zustande konnte ich den Schädel eines ausgewachsenen Hundes (Spitz) und eines Fuchses untersuchen. Bei beiden findet sich (Fig. 7, Taf. I) unter dem Canal. semicireul. sup. eine $5-6 \mathrm{Mm}$. weite rundliche Oeffnung, die in einen ebenso weiten und tiefen, von der Dura mater ausgekleideten und einen Anhang des kleinen Gehirns umfassenden Hoblraum führt. An den Schädeln anderer Hundearten aus der Sammlung (Mops, Pudel, Windhund) fand sich das gleiche Verhältniss vor. Eine ziemlich beträchtliche Grösse zeigte der Hohlraum bei einem Marder, den ich gleichfalls frisch zur Untersuchung bekam.

Von ungemein grossem Umfange erweist sich, wie $\mathrm{Cuvier}$ und Hyrtl übereinstimmend hervorheben, die Höble bei der Robbe. An dem wegen seiner Configuration sehr interessanten Felsenbeine derselben (grosse halbkugelförmige Bulla ossea mit engem äusseren Gehörgange) findet sich iiber und hinter dem Meat. auditor. intern. eine dreieckige Oeffnung mit etwas abgerundeten Winkeln, die an der Basis $>1_{12} \mathrm{Mm}$., in der Höhe $7 \mathrm{Mm}$. misst. Dieselbe führt in einen tiefen, fast um das Doppelte sich erweiternden Hohlraum, der nach hinten zu, gegen die hintere Pyramidenfläche, mit einer rundlichen, $3 \mathrm{Mm}$. weiten Oeffnung mündet (Fig. 8, Taf. I).

Aus der Ordnung der Beutelthiere stand mir nur der Schädel eines Känguruh zu Gebote. An ihm findet sich diese Höhle mit einer $4 \mathrm{Mm}$. weiten Oeffnung, aber nur geringer Tiefe unter dem oberen halbcirkelförmigen Kanale vor.

Die Ordnung der Nager lieferte mir ihre Repräsentanten im Biber, Hase, Kaninchen, Maus und Ratte zur Untersuchung. Bei allen diesen zeigt sich (vom Hasen sind die Grössenverhältnisse weiter oben schon angegeben, beim Kaninchen, als Fossa mastoidea von $\mathrm{Krause}^{1}$ ) bezeichnet, entbält sie den der Flocke

1) W. Krause, Anatomie des Kaninchens. S. 220. 
entsprechenden Lob. poster. cerebelli) die Grube ungemein geräumig und gross. Bei der Maus von einem Umfange (2 Mm. weit bei $6 \mathrm{Mm}$. Gesammtgrösse des Felsenbeines), der gegenüber den Grössenverhältnissen des ganzen Felsenbeines als ein sehr beträchtlicher angesehen werden muss.

Das Fehlen dieser Bildung bei den Ordnungen der Dickhäuter, Einbufer and Wiederkäuer, im ausgewachsenen Zustande sowohl als auch im fötalen, hat die Untersuchung der betreffenden Schädel, sowie solcher von Embryonen des Schweines, Rindes und Schafes ergeben.

Von grösster Wichtigkeit unter den Angaben $\mathrm{Hy}$ rtl's scheint mir diejenige zu sein, welche auf ein Verschwinden dieser Bildung mit dem vollendeten Wachsthume hinweist. Auch ich fand an einem sagittal durchschnittenen Schädel eines ausgewachsenen Exemplares von Simia satyrus der hiesigen Sammlung von genannter Grube beiderseits nach oben und hinten vom Meat. auditor. intern. nur noch eine feine, eingezogene Knochenöffnung vor, welche ganz derjenigen gleicht, die als Ueberrest der Fossa subarcuata, als Hiatus subarcuatus am Felsenbein des erwachsenen Menschen sich findet. Das Vorhandensein derselben bei einem jungen Exemplare von Simia satyrus hat, wie schon oben angegeben, Hyrtl hervorgehoben.

In Betreff des Gehirntheiles, der in diesen Hohlraum zu liegen kommt, haben wir nur die eine bestimmte Aeusserung Huxley's. Cuvier spricht von einer. Protuberanz des kleinen Gehirns, Hyrtl von gewissen flockenartig vereinigten Läppchen desselben; $\mathrm{Huxley}$ allein bezeichnet ibn als Flocculus, womit auch meine Beobachtungen an den frisch zur Untersuchung gekommenen Objecten ubereinstimmen.

Die Blutversorgung der diese Höhle auskleidenden Dura mater geschieht, wie ich an einem injicirten Kaninchenschädel gesehen habe, durch zwei ziemlich mächtige Aestchen der Art. mening. media, welche, sich vielfach verzweigend, an der vorderen und hinteren Umrandung des Bogens eintreten.

Versucht man es, die Angaben, welche die einzelnen Autoren uiber das Vorkommen der uns hier besehäftigenden Grube machen, uibersichtlich zusammenzustellen, so ergibt sich Folgendes:

In der Säugethierreihe fehlt die unter dem Can. semicircul. sup. sich eröffnende Grube vollständig in den Ordnungen der Fischsäuyethiere, Wiederküuer, Einhufer und Dickhäuter. Sie tritt vereinzell nur auf bei einigen Edentaten, häufiger werdend 
unter den Nagern und Beutlern, Fleisch- und Insektenfressern, ganz allgemein bei den Handflüglern und Vierhändern, bei welch letzteren sie sodann entweder während des ganzen Lebens sich erhält, oder, wie es beim Menschen der Fall ist, nur im jugendlichen Zustande sich findet, bei vollendeter Entwicking aber bis auf eine schwache Spur zurückgebildet erscheint.

Legt man sich die Frage vor, welches wohl die Verhältnisse sind, die in bestimmten Ordnungen der Säugethierreihe die uns beschäftigende Grube auftreten, in anderen wieder vollständig mangeln lassen, so könnten hier verschiedene Momente zu einer Erklärung herbeigezogen werden.

So war mir zu verschiedenen Malen bei der Betrachtung der Felsenbeine in den verschiedenen Thierschädeln die Stellung derselben aufgefallen, die Abweichung von der horizontalen Lage, die es beim Menschen einnimmt, so dass ich glaubte, vielleicht in dieser Richtung einen Zusammenhang zwischen der Stellung des Felsenbeines und dem Vorkommen der fraglichen Grube auffinden zu können. Genauere Nachforschungen an den Schädeln verschiedener Ordnungen und Pruffung der Angaben, die Köstlin hierüber macht, ergaben ein negatives Resultat. Kö stlin ${ }^{1}$ ) sagt über die Stellung des Felsenbeines im Schädel Folgendes : "Ausser dem Menschen, den beiden böchsten Affen und dem Elephanten dehnt sich bei keinem anderen Säugethiere das Felsenbein so überwiegend horizontal aus; bei den meisten schwankt es zwischen der Horizontalen und Senkrechten; nur bei den Nagern überwiegt die letztere Richtung entschieden und tritt da, wo das Felsenbein besonders gross ist, ganz rein auf." Gerade aber bei den Nagern findet sich die fragliche Grube in ziemlicher Häufigkeit gross und umfangreich vor, während sie beim Elephanten vollständig fehlt.

Dass auch nicht das Verhältniss der Bogengänge zu einander, respective der Neigungswinkel ihrer Ebenen und insbesondere der des oberen und hinteren für das Auftreten derselben in Betracht kommt, mag daraus hervorgehen, dass $\mathrm{Hyrtl}^{2}$ ), der eine Anzahl vergleichender Messungen hierüber vorgenommen hat, angibt, der Neigungswinkel der Bogenröhren sei nur ganz geringen Abweichungen unterworten, und komme eine auffallende Aenderung nur dem Lageverhältniss des äusseren zum hinteren Kanale $z u$, die jedoch für unseren Zweck gleichfalls ohne Bedentung sich erweist.

1) Köstlin a. a. O. S. 42 .

2) Hyrtl a. a. O. S. 108. 
Sucht man die Erklärung in der Weise, dass man annimmt, der Zweck der fraglichen Grube sei der, eine Verminderung der Masse des Felsenbeines, eine Raumerweiterung der Schädelhöhle herbeizuführen, für gewisse Partien des dort gelagerten kleinen Gehirns Raum zu schaffen, so möchte diese Dentung einigermaassen Bestätigung finden in den Angaben, die Köstlin ${ }^{1}$ ) grösstentheils im Anschlusse an Cuvier über die verschiedenen Dimensionen der Schädelhöhle macht. Die betreffenden Angaben lauten: „Fasst man die Schädelhöhle überhaupt ins Auge, so erscheint sie vorberrschend breit bei den Wiederkäuern, Dickhäutern, Monotremen und Cetaceen."

In diesen Ordnungen, wo also der Breitendurchmesser des Schädels ïberwiegt, fehlt, wie wir gesehen haben, die betreffende Bildung vollständig.

"Auf der anderen Seite, heisst es dann bei Köstlin weiter, überwiegt bei den Fleischfressern, Beutlern, Nagern und Zahnlosen die Länge. Von den Fleischfressern beginnt eine Reibe, welche durch die Halbaffen und Affen aufsteigt und bei mannigfachen leichten Schwankungen wesentlich durch eine Verminderung des Längsdurchmessers charakterisirt ist; sie endigt beim Menschen, welcher durch die Höhe und Breite seiner Schädelhöble wieder den Cetaceen näher gerückt ist."

In den genannten Ordnungen nun sehen wir die fragliche Grube erst vereinzelt, dann häufiger auftreten, bei den Halbaffen und Affen dann ganz allgemein werden, wobei sie jedoch bei den letzteren schon wieder an Bedeutung verliert, indem sie nur, bei gewissen Arten wenigstens, zur Zeit des noch unvollendeten Schädelwachsthumes sich vorfindet und nach Abschluss desselben bereits eine Riuckbildung erfahren hat.

Gehen wir nun zum Schlusse nochmals anf die Verhältnisse der Fossa subarcuata am embryonalen und kindlichen Schläfenbeine des Menschen über, so lassen sich dieselben in folgender Weise auffassen. Die Grube an der Cartilago petrosa, wie sie ron $\mathrm{Kölliker}$ beschrieben und abgebildet worden ist, stellt die erste embryonale Anlage jenes Zustandes dar, den wir als Enfoncement cerebellenx Cuvier's, cerebellar fossa Owen's, Fossa mastoidea Krause's, als weiten, von Gehirnmasse erfullten Hohlraum zwischen den drei Bogengängen bei einer grossen Anzahl von Säugethieren als constante Bildung vorfinden. Mit der wei-

1) Köstlin a. a. O. S. 53.

Archiv f. Ohrenheilkunde. XIX. Bd. 
teren Entwicklung dieser embryonalen Anlage kommt es nun beim Menschen zu einer in Bezug auf Form und Gestaltung mit jener im Thierreiche vorhandenen ganz gleichartigen Bildung, welche nur darin abweichend erscheint, dass sie keine Gehirnpartien in sich birgt und von der Dura mater vollständig erfüllt und nach aussen abgeschlossen ist, sowie dass sie nicht während des ganzen Lebens constant sich "erhält, sondern, wie dies auch schon bei gewissen, dem Menschen am nächsten stehenden Arten zu beobachten war, mit dem Abschlusse des Wachsthumes sich zurïckbildet.

Durch die zugleich mit der harten Hirnhaut in sie eindringenden Blutgefässe dient dieselbe zur Blutversorgung und Ernährung des Schläfenbeinknochens, namentlich jener um die Bogengänge und die Höhlen des Warzenfortsatzes gelegenen Partien, und erlangt dadurch ihre für das Gehörorgan und unter Umständen auch für das Leben wichtige und verhängnissvolle Bedeutung.

\section{Erklärung der Abbildungen \\ (Tafel I).}

Fig. 1-5. Die Fossa subarcuata des menschlichen Schläfenbeines in verschiedenen embryonalen Perioden. Fig. 1 in dreifacher Vergrösserung, die übrigen in natürlicher Grösse.

Fig. 1 aus dem 3.-4. Monate.

$=2==5 .-6 .=$

$=3==6 .-7 .=$

$=4==8$. $=$

$=5$ vom neugeborenen Kinde.

$=6$ Schläfenbein eines jungen Affen.

$=7$ Schläfenbein vom Hunde.

$=8$ Schläfenbein vom Seehunde.

F. s. Fossa subarcuata. M. i. Meatus auditor. internus.

F. m. Fossa mastoidea der Foss. subarcuata d. Menschen entsprechend.

(Tafel II.)

Fig. 1-3 Durchschnitte durch das Felsenbein eines 51/2 monatl. Embryos. Doppelte Vergrösserung. Fig. 1 gleich am Eingange in die Fossa subarcuata; Fig. 2 etwa in der Mitte, Figur 3 am Ende derselben. F. s. der durch die Fossa eindringende Strang von Bindegewebe; C. s. Durchschnitte der halbcirkelförmigen Kanäle mit ihrer knöchernen Umrandung; C. t. Cavum tympani.

Fig. 4-6 Durchschnitte durch das Felsenbein eines 6 monatl. Kindes. Gleichfalls doppelte Vergrösserung. Bezeichnung wie oben. A. m. Antrum mastoideum. Fig. 4 vor dem Eingange in die Fossa subarcuata; Fig. 5 Mitte; Fig. 6 gegen das Ende derselben.

Fig. 7 Durchschnitt durch die Fossa subarcuata eines 4 jährigen Kindes. 100 fache Vergrösserung. S. sp. Hohlräume der Subst. spong. des Knochens. $a b$ Gefässdurchschnitte; $a$ Vene, $b$ Arterie, $c$ in die Hohlräume eindringende Bindegewebsstreifen. 


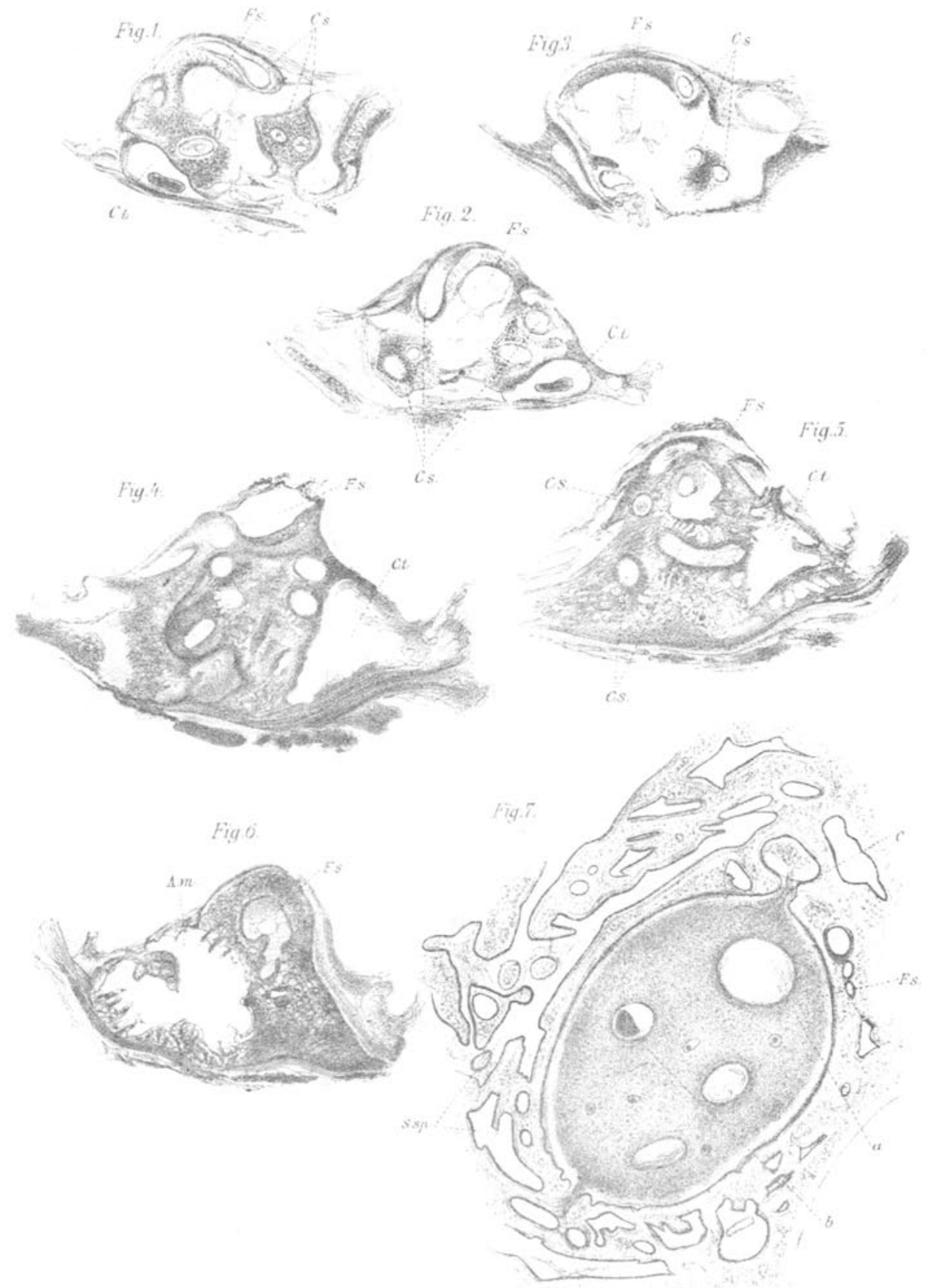

Wagenhauser, Anatonte a kndirhen Schafenbeins. 


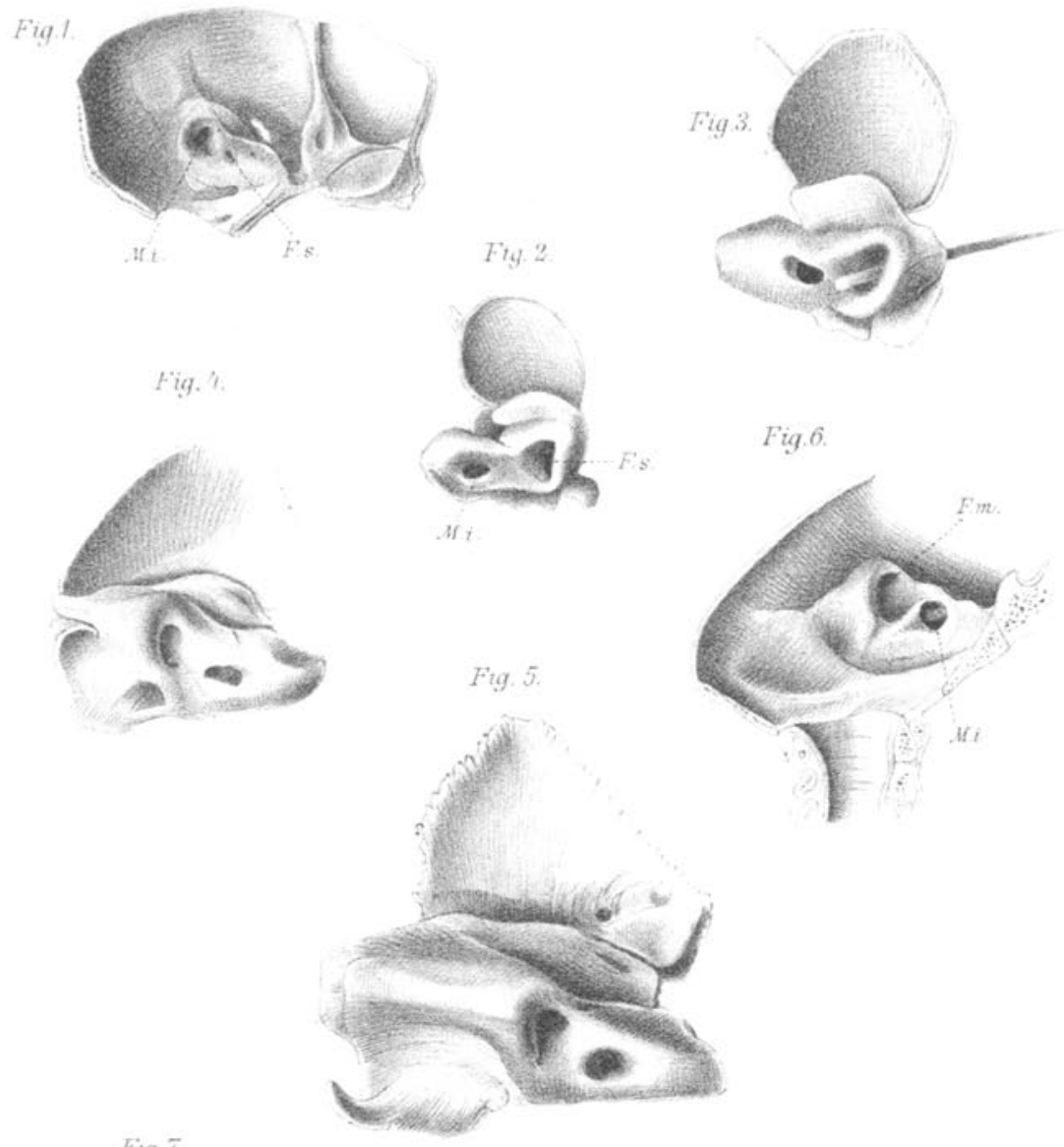

Fig $\pi$
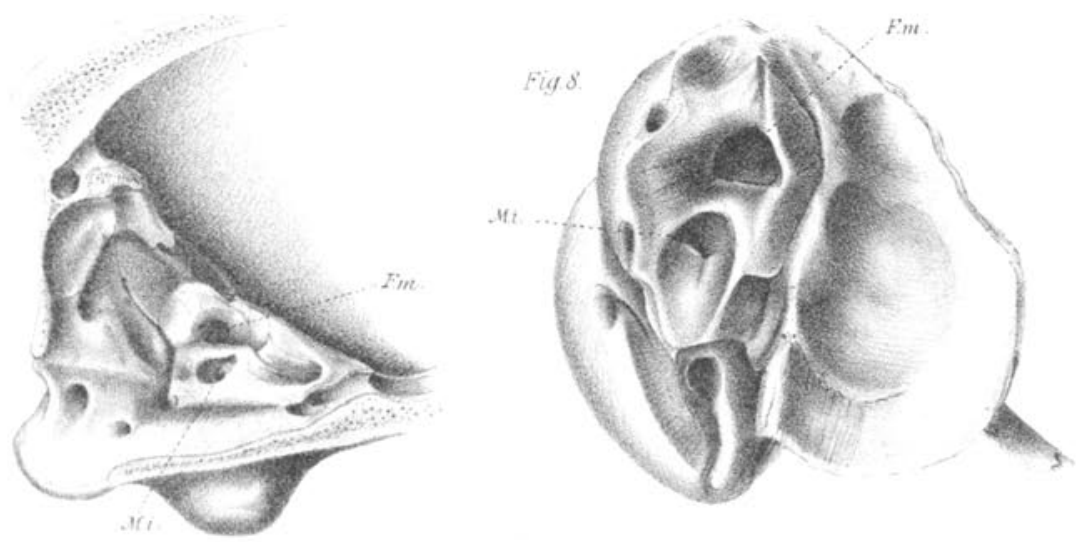

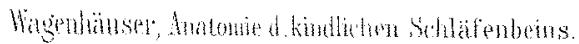



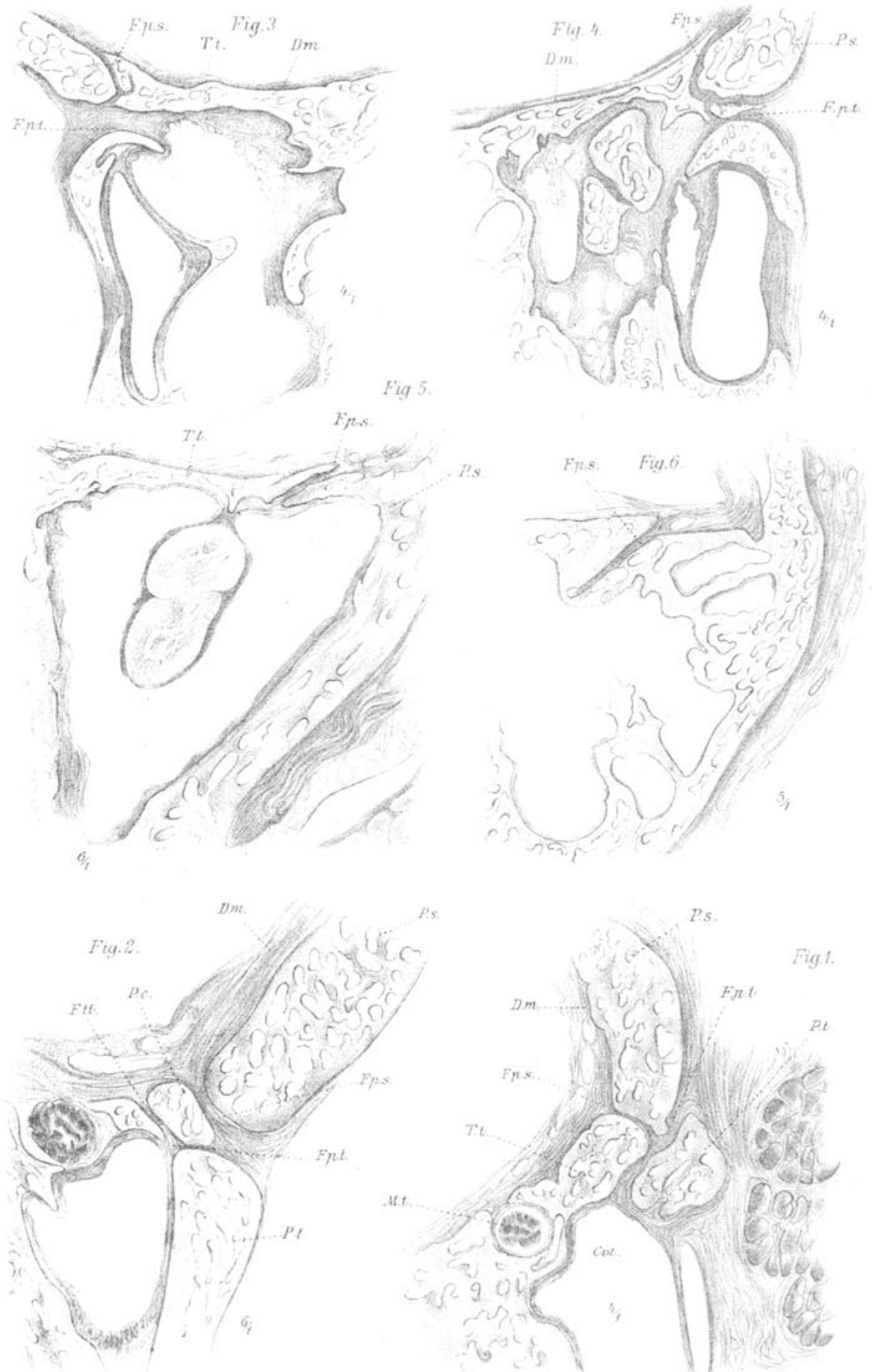

Ps:

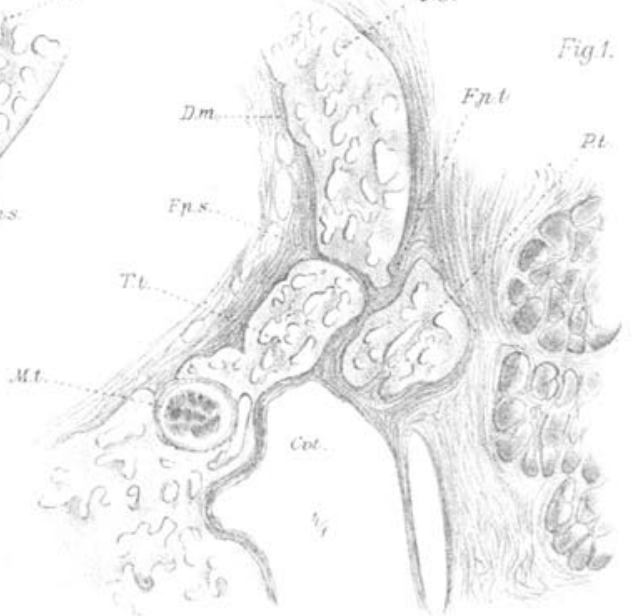

Wagenhäuser, Anatomie d. kudlufurn tohläfenbems.

FCWVogel in thist. 